\title{
Complete genome sequence of Thialkalivibrio versutus D301 isolated from Soda Lake in northern China, a typical strain with great ability to oxidize sulfide \\ Tingzhen Mu ${ }^{1,2}$, Jiemin Zhou ${ }^{3}$, Maohua Yang ${ }^{1}$, Jianmin Xing $^{1 *}$
}

${ }^{1}$ National Key Laboratory of Biochemical Engineering, Institute of Process Engineering, Chinese Academy of Sciences, P.O. Box 353, Beijing 100190, PR China. ${ }^{2}$ University of Chinese Academy of Sciences, Beijing 100049, PR China. ${ }^{3}$ State Key Laboratory of Mycology, Institute of Microbiology, Chinese Academy of Sciences, Beijing 100101, PR China.

*Corresponding author

E-mail address: jmxing@ipe.ac.cn

\begin{abstract}
Thioalkalivibrio versutus D301 isolated from Soda Lake is a haloalkaliphilic and obligated chemolithoautotrophic Gram- negative bacterium. The strain has a good adaption to hyperhaline and highly alkaline environment and a powerful sulfur-oxidizing ability. Here, we present the complete genome sequence of Thioalkalivibrio versutus D301, providing insights into the genomic basis of its effects and facilitating its application in microbial desulfurization.
\end{abstract}

\section{Introduction}

Thioalkalivibrio versutus D301, a haloalkaliphilic and obligated chemolithoautotrophic Gram-negative bacterium, was isolated from Soda Lake in the Inner Mongolia of China. T. versutus D301 belongs to the genus Thioalkalivibrio, in which there are only nine validly described species and many uncharacterized isolates so far. Thioalkalivibrio strains were slow-growing, high-yield microorganisms with low oxidation rate of sulfide and thiosulfate (Sorokin et al., 2003). The members of this genus can grow well in some extreme environment such as hypersaline (up to salt saturation) and alkaline (pH 7.5-10.5) (Muyzer et al., 2011a). Most of them can utilize $\mathrm{CO}_{2}$ as a carbon source and reduce inorganic sulfur compounds, such as sulfide, thiosulfate, element sulfur, sulfite, polythionates and so on, as an energy source. The members of the Thioalkalivibrio genus play an important role in the sulfur cycle on the earth, especially on the soda lakes. Moreover, Thioalkalivibrio species also have a great tendency to remove sulfide from wastewater and gas streams (van de Bosch et al., 2008). Van den Bosch (2008) verified that a domination of obligate chemolithoautotrophic sulfur-oxidizing bacteria (SOB) belonged to the genus Thioalkalivibrio in sulfide-oxidizing bioreactors, and T. versutus and T. jannaschii were core group when $\mathrm{pH}$ values reached 10. In order to understand the role of Thioalkalivibrio versutus D301 in the oxidation of sulfur compounds and its adapting ability to extreme alkaline and hypersaline conditions, we sequencedits complete genome.

The genome of Thioalkalivibrio versutus D301 was sequenced at Beijing Novogene Bioinformatics Technology Co.,Ltd. with massively parallel sequencing Illumina technology. Two DNA libraries were constructed: a paired-end library with an insert size of $500 \mathrm{bp}$ and a mate-pair library with an insert size of $5 \mathrm{~kb}$. The 500 bp library was sequenced using an Illumina MiSeq by PE300 strategy. A total of 1209 $\mathrm{Mb}$ filtered paired-end reads were obtained to reach a depth of 200-fold coverage of the genome with an Illumina MiSeq and HiSeq 2500 were obtained using the SOAPdenovo software. Gaps were closed by PCR and subsequent Sanger sequencing. Gene prediction was performed by GeneMarkS (Besemer et al., 2001). Genome annotation was performed using the PGAAP Pipeline (NCBI, 2010). Moreover, some software were used to predict the other elements in the genome, including rRNAmmer for rRNA (Lagesen et al., 2007), tRNAscan for tRNA (Lowe and Eddy, 1997), IslandPath-DIOMB for 
pathogenicity island (Hsiao et al., 2003), CRISPRFinder for CRISPR (Grissa et al., 2007) and so on.

The genome of Thioalkalivibrio versutus D301 was characterized by a circular chromosome of $2,969,361$ bp with a $66.03 \% \mathrm{G}+\mathrm{C}$ content without plasmids. The chromosome contains approximately 2,672 putative genes for coding protein, 45 tRNA-encoding genes and 1 rRNA operons (16S, 23S and 5S). (Table 1)

So far, four complete sequencing genomes of the Thioalkalivibrio genus have been published, but only two genomes from Thioalkalivibrio sulfidophilus HL-EbGr7 and Thioalkalivibrio.sp.K90mix were analyzed detailedly (Muyzer et al., 2011a; Muyzer et al., 2011b). The genome sequence of $T$. versutus D301 will certainly facilitate emerging new knowledge, developing genetic tools and speeding up its application in environmental protection.

\section{Nucleotide sequence accession number}

The complete genome sequence has been deposited in GenBank (GenBank ID: CP011367), and the genome project is uploaded in the Genome OnLine Database (GOLD ID: Gp0115210). The strain has been deposited in China General Microbiological Culture Collection Center (CGMCC No.8497). Also, the strain is available from Prof. Jianmin Xing (National Key Laboratory of Biochemical Engineering, Institute of Process Engineering, Chinese Academy of Sciences, Beijing, PR China).

\section{Acknowledgements}

This work was supported by the National Science Foundation of China (No.31370078), the National High Technology Research and Development Program of China (863 Project, No. 2011AA060904).

\section{References}

Besemer, J., Lomsadze, A., Borodovsky, M., 2001. GeneMarkS: a self-training method for prediction of gene starts in microbial genomes. Implications for finding sequence motifs in regulatory regions. Nucleic Acids Res. 29(12), 2607-2618.

Grissa, I., Vergnaud, G., Pourcel, C., 2007. CRISPRFinder: a web tool to identify clustered regularly interspaced short palindromic repeats. Nucleic Acids Res. (suppl 2), W52-W57.

Hsiao, W., Wan, I., Jones, S.J., Brinkman F.S.L., 2003. IslandPath: aiding detection of genomic islands in prokaryotes. Bioinformatics. 19(3), 418-420.

Lagesen, K., Hallin, P., Rødland, E.A., Stærfeldt, H., Rognes, T., Ussery, D. W., 2007. RNAmmer: consistent and rapid annotation of ribosomal RNA genes. Nucleic Acids Res. 35(9), 3100-3108.

Lowe, T.M., Eddy, S.R., 1997. tRNAscan-SE: a program for improved detection of transfer RNA genes in genomic sequence. Nucleic Acids Res. 25(5), 955-964.

Muyzer, G., Sorokin, D.Y., Mavromatis, K., Lapidus, A., Clum, A., Ivanova, N., et al., 2011a. Complete genome sequence of "Thialkalivibrio sulfidiphilus HL-EbGr7. Stand. Genomic. Sci. 4(1):23-35.

Muyzer, G., Sorokin, D.Y., Mavromatis, K., Lapidus, A., Foster, B., Sun, H., et al., 2011b. Complete genome sequence of Thialkalivibrio sp. K90mix. Stand. Genomic. Sci. 5(3), 341-355.

NCBI, 2010. NCBI Prokaryotic Genomes Automatic Annotation Pipeline (PGAAP), http://www.ncbi.nlm.nih.gov/genome/annotation prok/

Sorokin, D.Y., Banciu, H., van Loosdrecht, M., Kuenen, J.G., 2003. Growth physiology and competitive interaction of obligately chemolithoautotrophic, haloalkaliphilic, sulfur-oxidizing bacteria from soda lakes. Extremophiles. 7, 195-203.

van de Bosch, PLF., Sorokin, D.Y., Buisman, CJN., Janssen, AJH., 2008. The effect of pH on thiosulfate formation in a biotechnological process for the removal of hydrogen sulfide from gas streams. Environ. Sci. Technol. 42(7), 2637-2642. 
Table 1. Genome features of Thioalkalivibrio versutus D301.

\begin{tabular}{lr}
\hline Attribute & Value \\
\hline Genome Size(bp) & $2,969,361$ \\
DNA coding (bp) & $87.9 \%$ \\
DNA G+C (bp) & $66.03 \%$ \\
Replicons & 1 \\
Plasmids & 0 \\
Total genes & 2,761 \\
Protein coding genes & 2,672 \\
rRNA operons & 1 \\
tRNA genes & 45 \\
Pathogenicity islands & 5 \\
Prophage & 1 \\
CRISPR repeats & 2 \\
\hline
\end{tabular}

